\title{
Breaching the castle walls: hyaluronan depletion as a therapeutic approach to cancer therapy
}

\author{
H. Michael Shepard ${ }^{*}$ \\ Halozyme Therapeutics Inc., San Diego, CA, USA
}

Hyaluronan ( $\mathrm{HA})$ has many functions in the extracellular milieu of normal and diseased tissues. Disease-associated HA accumulation has been shown to predict a worsened prognosis in cancer patients, with tumors having a high-extracellular HA content (HA-high) being more aggressive than their HA-low counterparts. HA-high tumor aggressiveness is derived from the specialized biomechanical and molecular properties of the HA-based assembly of $\mathrm{HA}$ binding proteins and the growth-promoting factors that accumulate in it.

OPEN ACCESS

Edited by:

Rajeev K. Boregowda, Rutgers Cancer Institute of

New Jersey, USA

Reviewed by: Silvia Pastorekova,

Slovak Academy of Sciences,

Slovakia

Julie Valerie Decock

Qatar Biomedical Research Institute,

Qatar

*Correspondence:

H. Michael Shepard,

Halozyme Therapeutics Inc., 11388 Sorrento Valley Road, San

Diego, CA 92121, USA

mshepard@halozyme.com

Specialty section:

This article was submitted to Molecular and Cellular Oncology,

a section of the journal

Frontiers in Oncology

Received: 15 May 2015 Accepted: 10 August 2015 Published: 28 August 2015

Citation:

Shepard HM (2015) Breaching the castle walls: hyaluronan depletion as a therapeutic approach to cancer therapy.

Front. Oncol. 5:192.

doi: 10.3389/fonc.2015.00192
Biophysical characteristics of an HA-high tumor microenvironment include high tumor interstitial pressure, compression of tumor vasculature, and resulting tumor hypoxia. Within the tumor cell membrane, HA receptors, primarily CD44 and RHAMM, anchor the HA-high extracellular network. HA-CD44 association on the tumor cell surface enhances receptor tyrosine kinase activity to drive tumor progression and treatment resistance. Together, malignant cells in this HA-high matrix may evolve dependency on it for growth. This yields the hypothesis that depleting HA in HA-high tumors may be associated with a therapeutic benefit. A pegylated form of recombinant human hyaluronidase $\mathrm{PH} 2 \mathrm{O}$ (PEGPH20) has been deployed as a potential cancer therapeutic in HA-high tumors. PEGPH2O can collapse this matrix by degrading the HA-assembled tumor extracellular framework, leading to tumor growth inhibition, preferentially in HA-high tumors. Enzymatic depletion of HA by PEGPH2O results in re-expansion of the tumor vasculature, reduction in tumor hypoxia, and increased penetration of therapeutic molecules into the tumor. Finally, HA-depletion results in reduced signaling via CD44/RHAMM. Taken together, HA-depletion strategies accomplish their antitumor effects by multiple mechanisms that include targeting both biophysical and molecular signaling pathways. Ongoing clinical trials are examining the potential of PEGPH2O in combination with partner therapeutics in several cancers.

Keywords: hyaluronan, extracellular matrix, tumor microenvironment, hyaluronidase, treatment resistance, interstitial fluid pressure, PEGPH20

\section{Introduction}

Hyaluronan (HA) is a large, unbranched, glycosaminoglycan that consists of repeating disaccharides of D-glucuronic acid and $\mathrm{N}$-acetylglucosamine (1). HA is commonly referred to as a ubiquitous structural component of the extracellular, pericellular, and intracellular matrices, often with the function of lubricating surface-to-surface movement of joints or muscle (2). That characterization, although correct, does not convey the importance of the role that HA, together with its partner 
proteoglycans [the HA binding proteins (HABPs, or hyaladherins)], play in regulating cell behavior. HA in solution is polyanionic, conferring a largely negatively charged molecule in solution. Extensive hydration and the polyanionic nature of HA allow it to expand and occupy a large hydrodynamic volume $(3,4)$. High concentrations of HA, or HA combined with HABPs, creates a size-selective barrier in which small molecules can diffuse freely but larger molecules are partially or completely excluded (5). The biophysical properties of HA are exploited by tumor cells to create a sanctuary that is protective with respect to systemic therapies as well as host immune surveillance, as will be discussed below. Overall, the accumulation of HA in tumors can result in more aggressive malignancy (6-15). This review provides a brief overview of selected functions of HA and discusses the possible mechanisms through which accumulation of HA can make a tumor more aggressive and the mechanisms through which HA depletion can improve therapy of cancer.

\section{Hyaluronan: A Key Component of the Tumor Microenvironment and Promising Therapeutic Target}

\section{Hyaluronan and its Binding Partners}

The properties of HA that clearly affect tumor progression include its biophysical properties, which lead to accumulation of large amounts of water into the tumor microenvironment (TME) as a result of its hygroscopic nature as well as its net negative charge, which induces the molecule to take on an expanded volume of hydration (4). HA molecules within the TME absorb a substantial number of water molecules ( $\sim 15$ per disaccharide) causing the extracellular matrix (ECM) to swell, resulting in high tumor interstitial pressure (tIP), collapse of the tumor vasculature, and tumor hypoxia $(16,17)$. In addition, as HA is extruded into the TME by tumor cells, it also coordinates the assembly of a complex ECM through its ability to bind an array of HABPs (6, 18). Evidence from in vitro studies suggests that high expression of HAS3 induces abundant cell surface microvilli, which vastly increases the surface area of the tumor cell. Tumor cells within a tumor with high-HA accumulation (HA-high) are networked into the ECM by the HA receptors on their surface. The best characterized interaction is between HA and its principal receptor, CD44. HA in vitro forms three-dimensional pericellular coats and can also form cables that may be involved in cell-to-cell communication (19). The HA-high matrix provides scaffolding for many HABPs $(6,18,20)$. The matrix structural HABPs are primarily proteoglycans, and the most common side chains are composed of chondroitin sulfates $(6,18)$. Some HABPs [e.g., pentraxin-3, together with inter-alpha trypsin inhibitor and tumor necrosis factor-stimulated gene-6 (TSG-6)] serve to cross link the HAhigh tumor ECM (21). Higher levels of pentraxin-3 expression are associated with a more aggressive disease in pancreatic cancer patients (22). The chondroitin sulfates add to the elevated tIP because they coordinate water molecules like HA, and their dense negative charge can bind and store growth factors, which creates a reservoir of tumor-promoting molecules within the TME (23). However, in our work, the antitumor response to polyethylene glycol-conjugated (pegylated) recombinant human hyaluronidase
PH20 (PEGPH20) correlates very closely to the HA content of a tumor (24). The result of these interactions in an HA-high tumor is a multidimensional and gel-like structure comprising malignant cells, fibroblasts, and immune cells, all tied up together by HA and its binding partners in a setting of high tIP (Figure 1A). The high tIP characteristic of HA-high tumors may have other biomechanical signaling consequences promoting tumor progression $(25,26)$.

Aside from creating a unique TME structured around HA, cells that overproduce HA have additional properties. Recently, Tammi and colleagues described the formation of tumor cell membrane protrusions that accompany the overexpression of HAS3, one of three enzymes that synthesize and secrete HA (27). These microvilli, which can spontaneously break away from the tumor cells, retain the HAS3 enzyme and are coated in HA. Because they are coated with HA, they have the potential to bind/activate CD44/RHAMM on other cells, including stromal cells. Such membrane vesicles could be an important messaging system from a HA-high tumor that could affect the behavior of other cells, locally or systemically (28).

The HA-high tumor may have additional advantages. In particular, the HA pericellular coat can physically inhibit the ability of immune cells to form synapses and kill malignant cells in vitro (29). The extension of these in vitro findings to in vivo models has shown that in HA-high tumors, there is reduced access for either monoclonal antibody (mAb; trastuzumab) or natural killer (NK) cells (30). Depletion of HA from the tumor enables access and leads to improved efficacy, making HA a target for improving the efficacy of $\mathrm{mAb}$ or immune cell therapy of cancer (31).

Hyaluronan not only provides structural support to the tumor ECM but also interacts with cell surface receptors CD147/RHAMM and CD44. Both CD44 and RHAMM expression are tightly controlled by the wild-type p53 tumor suppressor gene $(32,33)$, provoking the hypothesis that their dysregulation contributes to tumor progression $(33,34)$. Activation, or amplified signaling, of multiple tyrosine kinases is mediated by HA binding to its receptors CD44 and RHAMM (33-36). Because elevated tyrosine kinase in tumor cells leads to resistance to macrophage killing, this could be an important pathway for HA/CD44 tumor cells to escape immune surveillance (37). In addition to the effects on tumor progression described above, further studies are underway to investigate the global reduction of growth factor signaling that is expected in tumor cells following depletion of HA $(36,38,39)$.

\section{Rationale for Targeting HA in Cancer}

Hyaluronan accumulates in the ECM of many solid tumors, and with a very high frequency $(87 \%)$ in pancreatic ductal adenocarcinoma (PDA) $(6,13,40)$. Several key factors provide a strong rationale for targeting HA in the TME (Figures 1A,B) $(16,17$, 40-45). Tumors that accumulate a relatively high amount of HA (the HA-high phenotype) have been shown to be more aggressive in mouse models and among cancer patients $(13,15,16$, 24). High HA production is sufficient for induction of epithelialto-mesenchymal transition (EMT) and acquisition of a highly malignant and migratory/invasive phenotype both in normal and transformed epithelial cells (46-48). In the genetically engineered Kras $^{L S L-G 12 D /+}{ }_{;} \operatorname{Trp} 53^{L S L-R 172 H /+}$;Cre (KPC) mouse model of PDA, 


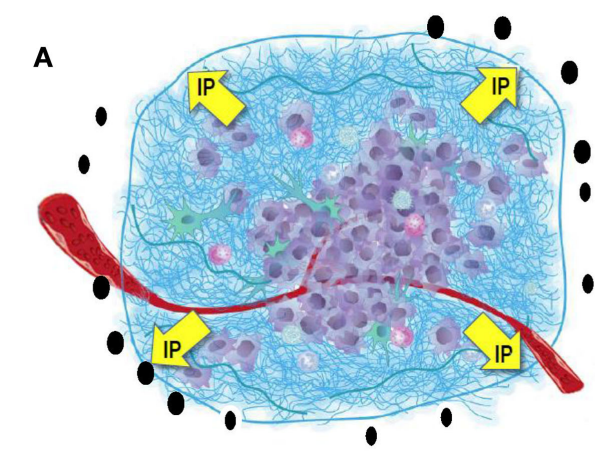

- High tumor interstitial pressure

- Compression of tumor blood vessels

- Hypoxia

- Protumorigenic growth factors and cytokines accumulate in the HA-rich tumor ECM

- Limited access of systemic therapies
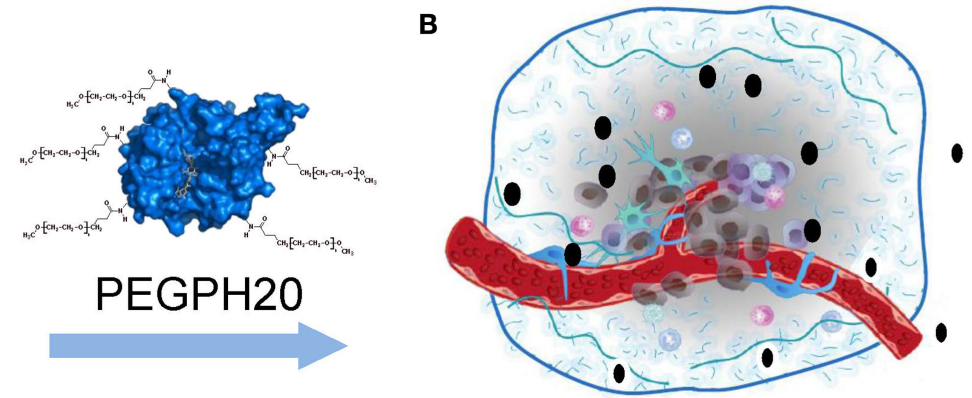

Tumor

- $\downarrow$ Tumor interstitial pressure (16)

- $\uparrow$ Vascular/tumor perfusion $(16,40)$

- $\downarrow$ Hypoxia (17)

- $\uparrow$ Access of systemic therapies to the tumor accompanied by increased efficacy $(16,40,41)$

Tumor Extracellular Matrix

- Depletion of HA, remodeling of the TEM $(16,17,40,41)$

- HMW HA L LMW HA; HA fragments released into the circulation as a result of systemic/tumor $\mathrm{HA}$ depletion (42), leading to decreased tumor IP

- Possible release of protumorigenic cytokines and growth factors

Malignant and Tumor Stromal Cells

- $\downarrow$ Activation of CD44/RHAMM signaling and $\downarrow$ tumor growth (43)

- $\downarrow$ EMT/metastasis (17)

- $\downarrow$ Possible that HA depletion may decrease available precursors for glycolysis thereby contributing to growth inhibition by PEGPH20 (44)
FIGURE 1 | The impact of HA depletion from a tumor with an HA-high phenotype. (A) An HA-high tumor, encompassed by a fibrous capsule. As HA accumulates in the tumor it adsorbs water, resulting in expansion of the tumor stroma, which is limited by the fibrous capsule, resulting in increased tumor interstitial pressure, collapse of tumor-associated vasculature, and other sequelae as shown. (B) After treatment with PEGPH20, high-molecular weight $\mathrm{HA}$ is degraded to fragments, which diffuse into newly expanded vasculature, resulting in a dose-dependent normalization of tumor interstitial pressure and other changes, which result in tumor growth inhibition and increased access to systemic therapies. Abbreviations: ECM, extracellular matrix; $\mathrm{HA}$,

hyaluronan; PEGPH20, pegylated recombinant human hyaluronidase; pO2, partial pressure of oxygen; VEGF, vascular endothelial growth factor. Figure adapted from Ref. (45). Data from Ref. (16, 17, 40-44).
HA deposition is seen early in the tumorigenesis process and persists during tumor progression and metastasis (41). Similar observations (conservation of the HA-high phenotype) have been reported in primary and secondary lesions from breast cancer patients (49).

\section{Breaching the HA Barrier}

Hyaluronidases function to degrade $\mathrm{HA}$ in a variety of tissue types and physiologic settings. The potential for HA to be an antitumor target was first pursued by Baumgartner and colleagues (50-52), who conducted several clinical studies with partially purified animal-derived $\mathrm{PH} 20$. The Baumgartner hypothesis was that HA in tumors impeded the tumor cell exposure to therapy. Although the investigators reported positive results from early trials, a randomized trial done in high-grade astrocytomas with combined chemotherapy and radiation therapy with and without hyaluronidase did not find an advantage in the $\mathrm{PH} 20$ group (51). Although additional clinical studies were not pursued at that time, our knowledge of the biology of HA has progressed significantly since these early investigations, and an image of how HA accumulation enhances tumor progression has emerged (previously discussed). In addition, the availability of purified recombinant human $\mathrm{PH} 20$ ( $\mathrm{rHuPH} 20)$ has enabled exploration of a means to use the human enzyme for chronic, systemic therapy (53). Both rHuPH20 and PEGPH20 were initially compared for clinical suitability through pharmacokinetic studies in mice, which showed that $\mathrm{rHuPH} 20$ had a very short half-life $(<3 \mathrm{~min})$, while PEGPH20 was shown to have an extended half-life $(10.3 \mathrm{~h})$ in vivo (16). The pegylated molecule has been taken forward in explorations of utility in cancer through both preclinical studies and clinical studies. Preclinical animal model studies using HAhigh and HA-low tumors were performed, all with the conclusion that tumors with the HA-high phenotype are more sensitive to PEGPH20 $(16,17,24)$.

The mechanism(s) through which HA-depletion results in tumor growth inhibition are still under investigation. Current data show that tumor perfusion by systemic therapies is increased following HA depletion by PEGPH20, resulting in a reversal of hypoxia and inducing other changes in the TME $(16,17)$. Accumulation of HA has been shown to be associated with loss 
of plasma membrane E-cadherin and $\beta$-catenin, suggesting disruption of adherens junctions, and increased potential for EMT, which is a predicate for the metastatic phenotype (54). PEGPH20 decreased the expression of hypoxia-related proteins and induced translocation of E-cadherin and $\beta$-catenin to the plasma membrane in vivo (17). Translocation of E-cadherin was also seen in tumors from a transgenic mouse model of pancreatic cancer and in a human non-small cell lung cancer sample from a patient treated with PEGPH20 (17). In conclusion, HA accumulation promotes tumorigenesis in multiple animal models and in many types of malignancies $(15-17,24,27,40,41)$. HA depletion reverses these changes and suppresses tumor growth. We hypothesize that the antitumor effects of PEGPH20 are likely due to these major changes in the TME. However, a direct effect on tumor cells is possible as a result of the generation of low-molecular weight (LMW)HA fragments that can compete with high-molecular weight (HMW)-HA for binding and activation of HABPs, including the HA receptors, CD44 and RHAMM (43). Literature describing the opposing roles of HMW-HA and LMW-HA is straightforward in cases in which there may be a high molar excess of LMW$\mathrm{HA}$, leading to antagonism of HMW-HA binding to hyaladherins and destabilization of the HA-mediated ECM. Other work suggests that accumulation of LMW-HA could lead to disruption of endothelial cell:cell interactions, and even to induction of inflammatory cytokines $(43,55)$. It is important to keep these proposed functions of LMW-HA in mind, as they could point to a toxicity resulting from systemic therapy with hyaluronidase. Studies in the KPC mouse model of PDA have shown that PEGPH20 treatment of tumor-bearing animals results in inhibition of tumor growth and a tumor-specific accumulation of chemotherapy (doxorubicin, gemcitabine), resulting in increased efficacy of these agents (40). Further studies have shown that increased tumor access to mAbs and immune cells can also occur following HA depletion. In vitro studies demonstrated that PEGPH20 treatment enhanced the access of trastuzumab and NK cells to HA-high tumors, and thereby increased both trastuzumaband NK-cell-mediated tumor growth inhibitions (31). The mechanism leading to this tumor-selective increase in perfusion is the rapid reversal of high tIP (Figure 2A) (16), which results in not only vessel reperfusion but also the formation of fenestrae in the tumor vascular endothelium (Figure 2B) (40). A similar mechanism may explain the increased accumulation of Salmonella vector encoding an immune-stimulating cargo in KPC tumor-bearing mice (56).

The depletion of HA from the tumor ECM is likely to have a role in the antitumor effects of other coadministered potential therapeutics. For example, inhibitors of HA synthase (4methylumbelliferone and methyl-beta-cyclodextrin) have been shown to have antitumor effects and can enhance the perfusion of chemotherapy into tumor vasculature $(28,57-59)$. Other agents that have been shown to affect tIP, but are not yet linked to HA loss, include the taxanes (60). Another recent report suggests that calcipotriol, a synthetic derivative of calcitriol $(1,25$ dihydroxyvitamin D3), can enhance the delivery of chemotherapy to pancreatic tumors through stromal remodeling (61). It is possible that the anti-inflammatory properties of calciferol are responsible for this result. Inflammatory signals are known to enhance HA synthesis, and result in HA accumulation and increased tIP (62). Suppression of inflammatory signals by calciferol is proposed to down-regulate the production of these mediators, which would
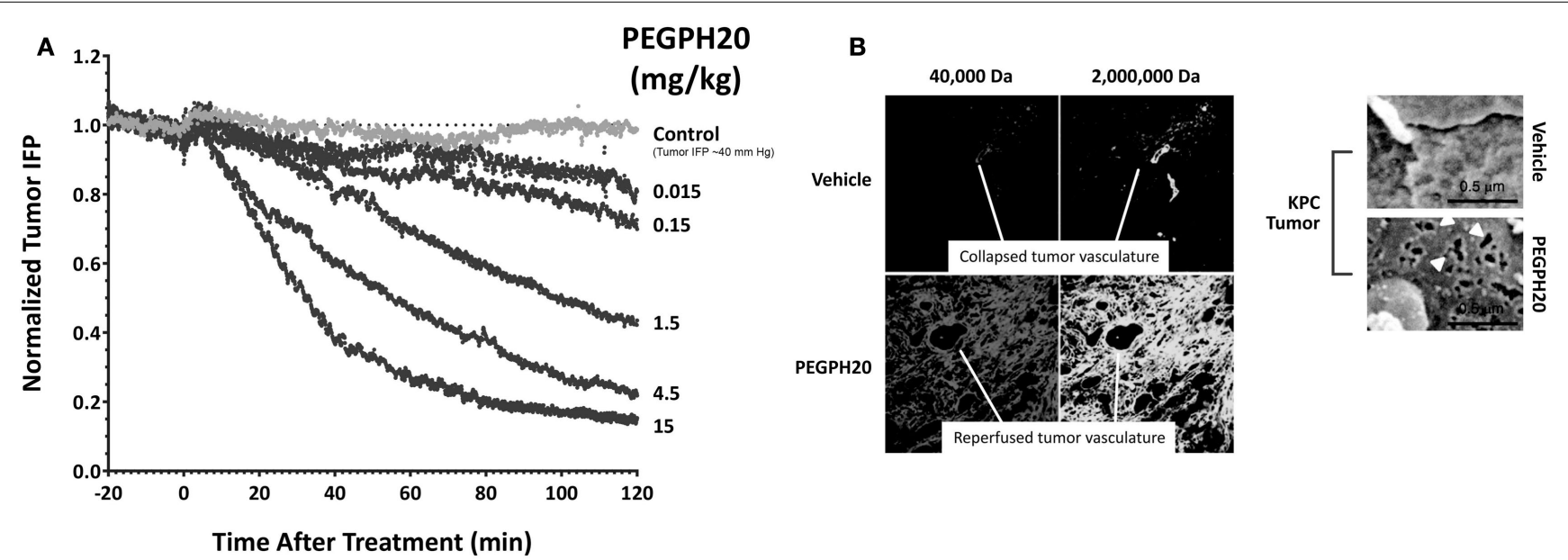

FIGURE 2 | Enzymatic HA depletion leads to normalization of tIP and normalization of tumor vasculature. (A) PEGPH20 rapidly normalizes tIP. Dose-dependent effect of PEGPH20 [0 (control), 0.015, $0.15,1.5,4.5,10$, and $15 \mathrm{mg} / \mathrm{kg}$ ] on tumor tIP in HA-high PC3 prostate tumors over a 2 -h period following intravenous administration (starting tIP $\sim 40 \mathrm{~mm} \mathrm{Hg}$ ). Abbreviations: HA, hyaluronan; IFP, interstitial fluid pressure; min, minutes; PEGPH20, polyethylene glycol-conjugated (pegylated) human hyaluronidase $\mathrm{PH} 20$; tIP, tumor interstitial pressure. Adapted with permission from Thompson et al. (16) (B) PEGPH20 treatment leads to vascular expansion and formation of endothelial fenestrae. Representative fluorescent images of KPC tumor from vehicle-treated (top left panels)

and PEGPH20-treated (bottom left panels) mice ( $n=4$ mice for each cohort). Scanning electron microscopy images of pancreatic blood vessels in KPC (upper two right panels) mice following treatment with either vehicle or PEGPH2O ( $n=4$ mice for each cohort) reveal endothelial fenestrations (white arrowheads) only in the tumor microvasculature. PEGPH20-treated KPC mice. Endothelia in the healthy pancreata of control PC mice are comparable to the untreated KPC tumor. Abbreviations: HA, hyaluronan; KPC, LSL-Kras ${ }^{G 12 D /+}$; LSLTrp53 $3^{R 172 H /+}$; Pdx-1-Cre; PC, LSL-Trp53R172H/+ ; Pdx-1-Cre; PEGPH20, polyethylene glycol-conjugated (pegylated) recombinant human hyaluronidase $\mathrm{PH} 20$. Adapted with permission from Jacobetz et al. (40). 
then lead to decreased HA accumulation. Although there are agents other than PEGPH20 that reduce tIP and may also lead to decreased HA accumulation, none have been shown to induce the rapid reperfusion and formation of vascular fenestrae observed with PEGPH20.

The role of hyaluronidases in tumor biology has long been an area of investigation. The $\mathrm{PH} 20$ hyaluronidase is unique in that it is active with respect to degrading $\mathrm{HA}$ at both low $\mathrm{pH}$ and neutral $\mathrm{pH}$. Other hyaluronidases (HYAL1, HYAL2, HYAL3, HYAL4) have been studied less extensively than PH20 $(63,64)$. From among these enzymes, HYAL1 has been proposed to promote tumorigenicity under certain conditions $(65,66)$. The mechanism(s) by which HYAL1 can promote tumorigenicity are still unclear. However, it is possible that the localized presence of HYAL1 in a tumor with the HA-high phenotype could be a mechanism to feed the increased dependence of tumor cells on glucose by facilitating the recycling of $\mathrm{HA}$ for cellular metabolism via the glycolysis pathway $(67,68)$, a process it may share with membrane associated HYAL2 (69). Therefore, long-term treatment with PEGPH20 might cause metabolic stress for tumor cells by depleting their supply of glucose precursors through removal of extracellular HA. In view of the reported tumor-promoting actions of Hyal1, it is also important to note that preclinical models have consistently shown a reduction in metastatic spread with PEGPH20 treatment $(6,41)$. These data serve to further highlight the differences between the various hyaluronidases and provide additional rationale for clinical development of PEGPH20. A summary of the proposed mechanisms of action for PEGPH20 enzyme therapy of cancer is shown in Figure 1B (16, 17, 40-45).

\section{Conclusion and Future Directions}

The correlation between excess HA accumulation in the stroma of solid tumors (including breast, prostate, lung, and pancreatic cancers) and poor prognosis and short survival has been demonstrated. Numerous preclinical studies have described a role for HA in the growth and metastasis of solid tumors. Potential

\section{References}

1. Meyer K, Palmer J. The polysaccharide of the vitreous humor. J Biol Chem (1934) 107:629-34.

2. Toole BP. Hyaluronan: from extracellular glue to pericellular cue. Nat Rev Cancer (2004) 4(7):528-39. doi:10.1038/nrc1391

3. Hascall V, Esko JD. Hyaluronan. In: Varki A, Cummings RD, Esko JD, Freeze $\mathrm{HH}$, Stanley P, Bertozzi CR, et al., editors. Essentials of Glycobiology, Chapter 15, 2nd ed. Cold Spring Harbor, NY: Cold Spring Harbor Laboratory Press (2009).

4. Cowman MK, Matsuoka S. Experimental approaches to hyaluronan structure. Carbohydr Res (2005) 340(5):791-809. doi:10.1016/j.carres.2005.01.022

5. Bookbinder LH, Hofer A, Haller MF, Zepeda ML, Keller GA, Lim JE, et al. A recombinant human enzyme for enhanced interstitial transport of therapeutics. J Control Release (2006) 114(2):230-41. doi:10.1016/j.jconrel.2006.05.027

6. Kultti A, Li X, Jiang P, Thompson CB, Frost GI, Shepard HM. Therapeutic targeting of hyaluronan in the tumor stroma. Cancers (Basel) (2012) 4(3):873-903. doi:10.3390/cancers4030873

7. Auvinen P, Tammi R, Parkkinen J, Tammi M, Agren U, Johansson R, et al. Hyaluronan in peritumoral stroma and malignant cells associates with breast cancer spreading and predicts survival. Am J Pathol (2000) 156(2):529-36. doi:10.1016/S0002-9440(10)64757-8 effects of HA accumulation include shielding cancer cells from immune cell attack and from antineoplastic therapies through a variety of mechanisms. Early phase clinical trials have demonstrated the benefits of adding PEGPH20 to chemotherapy for advanced pancreatic cancer. The results of a phase $1 \mathrm{~b}$ clinical trial with PEGPH20 together with gemcitabine in pancreatic cancer patients has shown promising signs of efficacy (70). A companion diagnostic [derived from the tumor necrosis factor-stimulated gene-6 (TSG6) HABP] is being co-developed with PEGPH20 (71), which should help to identify patients most likely to benefit from PEGPH20 therapy by assessing pretreatment biopsies and monitoring the effects of treatment. Ongoing clinical trials of PEGPH20 will provide a further understanding of the clinical benefit of this agent as an adjunct to standard therapies in advanced cancer. Two clinical trials to evaluate PEGPH20 are currently enrolling patients: a randomized phase $1 \mathrm{~B} / 2$ study of PEGPH20 plus modified FOLFIRINOX (leucovorin calcium, 5-fluorouracil, irinotecan hydrochloride, and oxaliplatin) versus modified FOLFIRINOX alone in patients with stage IV pancreatic cancer (72), and a randomized phase 2 study of PEGPH20 in combination with nabpaclitaxel and gemcitabine in patients with stage IV pancreatic cancer (73). These studies will further elucidate the benefits of PEGPH20 as an adjunct to anticancer therapies in patients with advanced pancreatic cancer, and studies are planned to investigate this strategy in other solid tumors. PEGPH20 is an important potential anticancer therapeutic because of its inherent antitumor activity, and its potential to be paired with other therapeutic modalities to achieve greater efficacy.

\section{Acknowledgments}

The author wishes to acknowledge valuable conversations with Dr. Curtis Thompson and support from Halozyme Therapeutics Inc. Financial support for medical editorial assistance was provided by Halozyme Therapeutics Inc. The author thanks Michael Hobert, Ph.D., ProEd Communications, Inc., for medical editorial assistance with this manuscript.

8. Anttila MA, Tammi RH, Tammi MI, Syrjanen KJ, Saarikoski SV, Kosma VM High levels of stromal hyaluronan predict poor disease outcome in epithelial ovarian cancer. Cancer Res (2000) 60(1):150-5.

9. Auvinen P, Tammi R, Kosma VM, Sironen R, Soini Y, Mannermaa A, et al. Increased hyaluronan content and stromal cell CD44 associate with HER2 positivity and poor prognosis in human breast cancer. Int J Cancer (2013) 132(3):531-9. doi:10.1002/ijc.27707

10. Boregowda RK, Appaiah HN, Siddaiah M, Kumarswamy SB, Sunila S, Thimmaiah $\mathrm{KN}$, et al. Expression of hyaluronan in human tumor progression. J Carcinog (2006) 5:2. doi:10.1186/1477-3163-5-2

11. Pirinen R, Tammi R, Tammi M, Hirvikoski P, Parkkinen JJ, Johansson R, et al. Prognostic value of hyaluronan expression in non-small-cell lung cancer: increased stromal expression indicates unfavorable outcome in patients with adenocarcinoma. Int J Cancer (2001) 95(1):12-7. doi:10.1002/10970215(20010120)95:1<12::AID-IJC1002>3.3.CO;2-5

12. Ropponen K, Tammi M, Parkkinen J, Eskelinen M, Tammi R, Lipponen P, et al. Tumor cell-associated hyaluronan as an unfavorable prognostic factor in colorectal cancer. Cancer Res (1998) 58(2):342-7.

13. Sironen RK, Tammi M, Tammi R, Auvinen PK, Anttila M, Kosma VM. Hyaluronan in human malignancies. Exp Cell Res (2011) 317(4):383-91. doi:10.1016/j. yexcr.2010.11.017 
14. Theocharis AD, Skandalis SS, Tzanakakis GN, Karamanos NK. Proteoglycans in health and disease: novel roles for proteoglycans in malignancy and their pharmacological targeting. FEBS J (2010) 277(19):3904-23. doi:10.1111/j.17424658.2010.07800.x

15. Whatcott CJ, Diep CH, Jiang P, Watanabe A, LoBello J, Sima C, et al. Desmoplasia in primary tumors and metastatic lesions of pancreatic cancer. Clin Cancer Res (2015) 21(15):3561-8. doi:10.1158/1078-0432.CCR-14-1051

16. Thompson CB, Shepard HM, O'Connor PM, Kadhim S, Jiang P, Osgood RJ, et al. Enzymatic depletion of tumor hyaluronan induces antitumor responses in preclinical animal models. Mol Cancer Ther (2010) 9(11):3052-64. doi:10.1158/ 1535-7163.MCT-10-0470

17. Kultti A, Zhao C, Singha NC, Zimmerman S, Osgood RJ, Symons R, et al. Accumulation of extracellular hyaluronan by hyaluronan synthase 3 promotes tumor growth and modulates the pancreatic cancer microenvironment. Biomed Res Int (2014) 2014:817613. doi:10.1155/2014/817613

18. Day AJ, Prestwich GD. Hyaluronan-binding proteins: tying up the giant. J Biol Chem (2002) 277(7):4585-8. doi:10.1074/jbc.R100036200

19. Rilla K, Siiskonen H, Tammi M, Tammi R. Hyaluronan-coated extracellular vesicles - a novel link between hyaluronan and cancer. Adv Cancer Res (2014) 123:121-48. doi:10.1016/B978-0-12-800092-2.00005-8

20. Dicker KT, Gurski LA, Pradhan-Bhatt S, Witt RL, Farach-Carson MC, Jia X. Hyaluronan: a simple polysaccharide with diverse biological functions. Acta Biomater (2014) 10(4):1558-70. doi:10.1016/j.actbio.2013.12.019

21. Baranova NS, Inforzato A, Briggs DC, Tilakaratna V, Enghild JJ, Thakar D, et al. Incorporation of pentraxin 3 into hyaluronan matrices is tightly regulated and promotes matrix cross-linking. J Biol Chem (2014) 289(44):30481-98. doi:10. 1074/jbc.M114.568154

22. Kondo S, Ueno H, Hosoi H, Hashimoto J, Morizane C, Koizumi F, et al. Clinical impact of pentraxin family expression on prognosis of pancreatic carcinoma. $\mathrm{Br}$ J Cancer (2013) 109(3):739-46. doi:10.1038/bjc.2013.348

23. Lim JJ, Temenoff JS. The effect of desulfation of chondroitin sulfate on interactions with positively charged growth factors and upregulation of cartilaginous markers in encapsulated MSCs. Biomaterials (2013) 34(21):5007-18. doi:10. 1016/j.biomaterials.2013.03.037

24. Jiang P, Li X, Thompson CB, Huang Z, Araiza F, Osgood R, et al. Effective targeting of the tumor microenvironment for cancer therapy. Anticancer Res (2012) 32(4):1203-12.

25. Provenzano PP, Inman DR, Eliceiri KW, Keely PJ. Matrix density-induced mechanoregulation of breast cell phenotype, signaling and gene expression through a FAK-ERK linkage. Oncogene (2009) 28(49):4326-43. doi:10.1038/ onc. 2009.299

26. Kumar S, Weaver VM. Mechanics, malignancy, and metastasis: the force journey of a tumor cell. Cancer Metastasis Rev (2009) 28(1-2):113-27. doi:10.1007/ s10555-008-9173-4

27. Tammi RH, Kultti A, Kosma VM, Pirinen R, Auvinen P, Tammi MI. Hyaluronan in human tumors: pathobiological and prognostic messages from cellassociated and stromal hyaluronan. Semin Cancer Biol (2008) 18(4):288-95. doi:10.1016/j.semcancer.2008.03.005

28. Nagy N, Kuipers HF, Frymoyer AR, Ishak HD, Bollyky JB, Wight TN, et al. 4-methylumbelliferone treatment and hyaluronan inhibition as a therapeutic strategy in inflammation, autoimmunity, and cancer. Front Immunol (2015) 6:123. doi:10.3389/fimmu.2015.00123

29. McBride WH, Bard JB. Hyaluronidase-sensitive halos around adherent cells. Their role in blocking lymphocyte-mediated cytolysis. J Exp Med (1979) 149(2):507-15.

30. Varadi T, Mersich T, Auvinen P, Tammi R, Tammi M, Salamon F, et al. Binding of trastuzumab to ErbB2 is inhibited by a high pericellular density of hyaluronan. J Histochem Cytochem (2012) 60(8):567-75. doi:10.1369/ 0022155412448070

31. Singha NC, Nekoroski T, Zhao C, Symons R, Jiang P, Frost GI, et al. Tumorassociated hyaluronan limits efficacy of monoclonal antibody therapy. $\mathrm{Mol}$ Cancer Ther (2015) 14(2):523-32. doi:10.1158/1535-7163.MCT-14-0580

32. Sohr S, Engeland K. RHAMM is differentially expressed in the cell cycle and downregulated by the tumor suppressor p53. Cell Cycle (2008) 7(21):3448-60. doi:10.4161/cc.7.21.7014

33. Godar S, Ince TA, Bell GW, Feldser D, Donaher JL, Bergh J, et al. Growth-inhibitory and tumor-suppressive functions of p53 depend on its repression of CD44 expression. Cell (2008) 134(1):62-73. doi:10.1016/j.cell. 2008.06.006
34. Ponta H, Sherman L, Herrlich PA. CD44: from adhesion molecules to signalling regulators. Nat Rev Mol Cell Biol (2003) 4(1):33-45. doi:10.1038/nrm1004

35. Grass GD, Dai L, Qin Z, Parsons C, Toole BP. CD147: regulator of hyaluronan signaling in invasiveness and chemoresistance. Adv Cancer Res (2014) 123:351-73. doi:10.1016/B978-0-12-800092-2.00013-7

36. Toole BP. Hyaluronan-CD44 interactions in cancer: paradoxes and possibilities. Clin Cancer Res (2009) 15(24):7462-8. doi:10.1158/1078-0432.CCR-09-0479

37. Hudziak RM, Lewis GD, Shalaby MR, Eessalu TE, Aggarwal BB, Ullrich A, et al. Amplified expression of the HER2/ERBB2 oncogene induces resistance to tumor necrosis factor alpha in NIH 3T3 cells. Proc Natl Acad Sci U S A (1988) 85(14):5102-6. doi:10.1073/pnas.85.14.5102

38. Simpson RM, Wells A, Thomas D, Stephens P, Steadman R, Phillips A. Aging fibroblasts resist phenotypic maturation because of impaired hyaluronandependent CD44/epidermal growth factor receptor signaling. Am J Pathol (2010) 176(3):1215-28. doi:10.2353/ajpath.2010.090802

39. Slomiany MG, Dai L, Tolliver LB, Grass GD, Zeng Y, Toole BP. Inhibition of functional hyaluronan-CD44 interactions in CD133-positive primary human ovarian carcinoma cells by small hyaluronan oligosaccharides. Clin Cancer Res (2009) 15(24):7593-601. doi:10.1158/1078-0432.CCR-09-2317

40. Jacobetz MA, Chan DS, Neesse A, Bapiro TE, Cook N, Frese KK, et al. Hyaluronan impairs vascular function and drug delivery in a mouse model of pancreatic cancer. Gut (2013) 62(1):112-20. doi:10.1136/gutjnl-2012-302529

41. Provenzano PP, Cuevas C, Chang AE, Goel VK, Von Hoff DD, Hingorani SR. Enzymatic targeting of the stroma ablates physical barriers to treatment of pancreatic ductal adenocarcinoma. Cancer Cell (2012) 21(3):418-29. doi:10. 1016/j.ccr.2012.01.007

42. Hingorani SR, Harris WP, Beck JT, Berdov BA, Wagner SA, Pshevlotsky EM, et al. Exploratory biomarker results from early investigation of PEGPH20 in combination with gemcitabine in patients with pancreatic cancer. Presented at the American Society of Clinical Oncology Gastrointestinal Symposium; 2015 January 15-17. San Francisco, CA (2015). Abstract 300.

43. Misra S, Hascall VC, Markwald RR, Ghatak S. Interactions between hyaluronan and its receptors (CD44, RHAMM) regulate the activities of inflammation and cancer. Front Immunol (2015) 6:201. doi:10.3389/fimmu.2015.00201

44. Thomas N, Waltham M, Udabage L, Brown T. Hyaluronan metabolism enhances the Warburg effect and provides an alternate energy source in the breast cancer microenvironment. Presented at the American Association for Cancer Research Annual Meeting; 2010 April 17-21. Washington, DC (2010). Abstract 58.

45. Ramanathan R, Infante JR, Rosen L, Von Hoff D, Dychter S, Shepard HM, et al. Targeting hyaluronan in tumor stroma: interim translational \& biomarker evaluations of pegylated hyaluronidase (PEGPH20) in patients with advanced solid tumors. Presented at the EORTC-NCI-ASCO Annual Meeting on Molecular Markers in Cancer; 2011 October 27-29. Brussels (2011). Abstract 60.

46. Jacobson A, Rahmanian M, Rubin K, Heldin P. Expression of hyaluronan synthase 2 or hyaluronidase 1 differentially affect the growth rate of transplantable colon carcinoma cell tumors. Int J Cancer (2002) 102(3):212-9. doi:10.1002/ijc. 10683

47. Li Y, Heldin P. Hyaluronan production increases the malignant properties of mesothelioma cells. Br J Cancer (2001) 85(4):600-7. doi:10.1054/bjoc.2001. 1922

48. Zoltan-Jones A, Huang L, Ghatak S, Toole BP. Elevated hyaluronan production induces mesenchymal and transformed properties in epithelial cells. J Biol Chem (2003) 278(46):45801-10. doi:10.1074/jbc.M308168200

49. Jadin L, Pastorino S, Symons R, Nomura N, Jiang P, Juarez T, et al. Hyaluronan expression in primary and secondary brain tumors. Ann Transl Med (2015) 3(6):80. doi:10.3978/j.issn.2305-5839.2015.04.07

50. Pillwein K, Fuiko R, Slavc I, Czech T, Hawliczek G, Bernhardt G, et al. Hyaluronidase additional to standard chemotherapy improves outcome for children with malignant brain tumors. Cancer Lett (1998) 131(1):101-8. doi: 10.1016/S0304-3835(98)00205-5

51. Baumgartner G, Gomar-Hoss C, Sakr L, Ulsperger E, Wogritsch C. The impact of extracellular matrix on the chemoresistance of solid tumors - experimental and clinical results of hyaluronidase as additive to cytostatic chemotherapy. Cancer Lett (1998) 131(1):85-99. doi:10.1016/S0304-3835(98)00204-3

52. Klocker J, Sabitzer H, Raunik W, Wieser S, Schumer J. Hyaluronidase as additive to induction chemotherapy in advanced squamous cell carcinoma of the head and neck. Cancer Lett (1998) 131(1):113-5. doi:10.1016/S0304-3835(98) 00207-9 
53. Frost GI. Recombinant human hyaluronidase (rHuPH20): an enabling platform for subcutaneous drug and fluid administration. Expert Opin Drug Deliv (2007) 4(4):427-40. doi:10.1517/17425247.4.4.427

54. Scheel C, Weinberg RA. Cancer stem cells and epithelial-mesenchymal transition: concepts and molecular links. Semin Cancer Biol (2012) 22(5-6):396-403. doi:10.1016/j.semcancer.2012.04.001

55. Singleton PA. Hyaluronan regulation of endothelial barrier function in cancer. Adv Cancer Res (2014) 123:191-209. doi:10.1016/B978-0-12-800092-2. 00007-1

56. Manuel E, Chen J, D’Apuzzo M, Lampa M, Kaltcheva T, Thompson C, et al. Salmonella-based therapy targeting indoleamine 2,3-dioxygenase coupled with enzymatic depletion of tumor hyaluronan induces complete regression of aggressive pancreatic tumors. Cancer Immunol Res (2015). doi:10.1158/23266066.CIR-14-0214

57. Nakazawa H, Yoshihara S, Kudo D, Morohashi H, Kakizaki I, Kon A, et al. 4-methylumbelliferone, a hyaluronan synthase suppressor, enhances the anticancer activity of gemcitabine in human pancreatic cancer cells. Cancer Chemother Pharmacol (2006) 57(2):165-70. doi:10.1007/s00280-005-0016-5

58. Piccioni F, Fiore E, Bayo J, Atorrasagasti C, Peixoto E, Rizzo M, et al. 4Methylumbelliferone inhibits hepatocellular carcinoma growths by decreasing IL-6 production and angiogenesis. Glycobiology (2015) 25(8):825-35. doi:10. 1093/glycob/cwv023

59. Kultti A, Karna R, Rilla K, Nurminen P, Koli E, Makkonen KM, et al. Methyl-beta-cyclodextrin suppresses hyaluronan synthesis by down-regulation of hyaluronan synthase 2 through inhibition of Akt. J Biol Chem (2010) 285(30):22901-10. doi:10.1074/jbc.M109.088435

60. Griffon-Etienne G, Boucher Y, Brekken C, Suit HD, Jain RK. Taxaneinduced apoptosis decompresses blood vessels and lowers interstitial fluid pressure in solid tumors: clinical implications. Cancer Res (1999) 59(15): 3776-82.

61. Sherman MH, Yu RT, Engle DD, Ding N, Atkins AR, Tiriac H, et al. Vitamin D receptor-mediated stromal reprogramming suppresses pancreatitis and enhances pancreatic cancer therapy. Cell (2014) 159(1):80-93. doi:10.1016/j. cell.2014.08.007

62. Vigetti D, Genasetti A, Karousou E, Viola M, Moretto P, Clerici M, et al. Proinflammatory cytokines induce hyaluronan synthesis and monocyte adhesion in human endothelial cells through hyaluronan synthase 2 (HAS2) and the nuclear factor-kappaB (NF-kappaB) pathway. J Biol Chem (2010) 285(32):24639-45. doi:10.1074/jbc.M110.134536

63. Csoka AB, Frost GI, Stern R. The six hyaluronidase-like genes in the human and mouse genomes. Matrix Biol (2001) 20(8):499-508. doi:10.1016/S0945053X(01)00172-X
64. Stern R. Hyaluronidases in cancer biology. Semin Cancer Biol (2008) 18(4):275-80. doi:10.1016/j.semcancer.2008.03.017

65. Nikitovic D, Kouvidi K, Voudouri K, Berdiaki A, Karousou E, Passi A, et al. The motile breast cancer phenotype roles of proteoglycans/glycosaminoglycans. Biomed Res Int (2014) 2014:124321. doi:10.1155/2014/124321

66. Lokeshwar VB, Mirza S, Jordan A. Targeting hyaluronic acid family for cancer chemoprevention and therapy. Adv Cancer Res (2014) 123:35-65. doi:10.1016/ B978-0-12-800092-2.00002-2

67. Roden L, Campbell P, Fraser JR, Laurent TC, Pertoft H, Thompson JN. Enzymic pathways of hyaluronan catabolism. Ciba Found Symp (1989) 143:60-76.

68. Ganapathy-Kanniappan S, Geschwind JF. Tumor glycolysis as a target for cancer therapy: progress and prospects. Mol Cancer (2013) 12:152. doi:10.1186/14764598-12- 152

69. Harada H, Takahashi M. CD44-dependent intracellular and extracellular catabolism of hyaluronic acid by hyaluronidase-1 and -2. J Biol Chem (2007) 282(8):5597-607. doi:10.1074/jbc.M608358200

70. Hingorani SR, Harris WP, Beck JT, Berdov BA, Wagner SA, Pshevlotsky EM, et al. A phase Ib study of gemcitabine plus PEGPH20 (pegylated recombinant human hyaluronidase) in patients with stage IV previously untreated pancreatic cancer. Presented at the American Society of Clinical Oncology Annual Meeting; 2013 May 31-June 4. Chicago, IL (2013). Abstract 4010.

71. Jadin L, Huang L, Zhao Q, Wei G, Shepard M, Gelb A, et al. Development of a Companion Diagnostic for Pegylated Recombinant Human PH20. Boston, MA: EORTC (2013) Abstract MC13-0055.

72. S1313, a Phase IB/II Randomized Study of Modified FOLFIRINOX + Pegylated Recombinant Human Hyaluronidase (PEGPH20) Versus Modified FOLFIRINOX Alone in Patients with Good Performance Status Metastatic Pancreatic Adenocarcinoma. Accessed from https://clinicaltrials.gov/ct2/show/NCT01959139

73. A Phase 2, Randomized, Multicenter Study of PEGPH20 (PEGylated Recombinant Human Hyaluronidase) Combined with Nab-Paclitaxel Plus Gemcitabine Compared with Nab-Paclitaxel Plus Gemcitabine in Subjects with Stage IV Previously Untreated Pancreatic Cancer. Accessed from: http://clinicaltrials.gov/ct2/ show/NCT01839487

Conflict of Interest Statement: Dr. H. Michael Shepard is an employee of Halozyme Therapeutics Inc.

Copyright (C) 2015 Shepard. This is an open-access article distributed under the terms of the Creative Commons Attribution License (CC BY). The use, distribution or reproduction in other forums is permitted, provided the original author(s) or licensor are credited and that the original publication in this journal is cited, in accordance with accepted academic practice. No use, distribution or reproduction is permitted which does not comply with these terms. 


\section{Appendix}

\section{Key Concepts}

Hyaluronan (HA)

Also known as hyaluronic acid, HA is a large, unbranched, glycosaminoglycan that consists of repeating disaccharides of $\mathrm{D}$ glucuronic acid and $\mathrm{N}$-acetylglucosamine, and is a key polysaccharide component of the ECM. Excess accumulation of HA in the tumor stroma is associated with poor prognosis and short survival.

\section{The Tumor Cell Sanctuary}

Cancers with the HA-high phenotype also have high tIP, which leads to tumor vascular collapse, hypoxia, and inherent drug resistance. Elevated tIP is also associated with increased metastatic capability. The presence of an HA-coat around tumor cells leads to activation of the CD44 receptor, and likely RHAMM, which enhances tumor growth, drug resistance, and metastasis.

\section{Depletion of HA has an Effect on the Tumorigenic Properties of an HA-High Tumor}

Several agents decrease HA from the tumor ECM. 4methylumbelliferone and methyl-beta-cyclodextrin inhibit HA synthesis, and PEGPH20 directly depletes HA. The fact that all three agents selectively inhibit tumor growth in HA-high tumors supports the validity of $\mathrm{HA}$ as a target in HA-high cancers.

\section{HA Depletion from a Tumor with the HA-High Phenotype Inhibits Tumor Growth by Several Mechanisms}

(A) Reversal of high tIP inhibits tumor cell EMT; (B) Reperfusion of the tumor by systemic chemotherapy, mAb therapy, and immune cell therapy; (C) Blocks CD44-associated signaling; (D) Reduced tyrosine kinase signaling increases sensitivity to immune surveillance and inhibits tumor growth and metastasis. 